\title{
Aknowledgment of Ad Hoc Reviewers
}

Emotion extends its thanks to the following individuals who contributed their time and expertise to the journal by serving as ad hoc reviewers for manuscripts submitted from September 2016 to September 2017.

Marije aan het Rot

Luigi Acerbi

Gabrielle Adams

Leah Adams

Lara Aknin

Evin Aktar

Theodore Alexopoulos

Sara Alger

Krypotos Angelos-Miltiadis

Oriana Aragon

Thomas Armstrong

Karen M. Arnell

Joel Aronoff

Dilbur Arsiwalla

Yoni Ashar

Chris Askew

Anu Asnaani

Sukriye Sinem Atakan

Anthony P. Atkinson

Elizabeth J. Austin

Eva Bänninger-Huber

Yoav Bar-Anan

Bruce D. Bartholow

Monica Bartlett

Brock Bastian

Jean-Yves Baudouin

Nicola Baumann

Sarah Bayless

Daniel Beal

Genna Bebko

D. Vaughn Becker

Lane Beckes

Tom Beesley

David Beltran

Nick Berggren

Christopher Berghoff

Amit Bernstein

Sara Berzenski

Brandy Bessette-Symons

B. Ann Bettencourt

Erik Bijleveld

Jeffrey Birk

Christophe Blaison

Shannon Blakey

Matthew Boden

Ryan Bogdan

Vanessa Bohns

Michael Boiger

George A. Bonanno

Mario Bonato

Sarah Bookbinder

Thomas Boone

Jessica L. Borelli

Alexia Bourgeois

Julienne Bower

Daniel Bradford

William Brady
Chris R. Brewin

David Bridgett

Margaret J. Briggs-Gowan

Kirk Brown

Stephanie Brown

Celia Brownell

Jazmin Brown-Iannuzzi

Emile Bruneau

Greg Bryant

Ross W. Buck

Erin Buckels

Jeni Burnette

Shannon Burns

Michael A. Busseri

Emily A. Butler

Lauren M. Bylsma

Roberto Caldara

Catherine Caldwell-Harris

Manuel G. Calvo

Rafaela Campagnoli

Laura Campbell-Sills

Linda A. Camras

Arnie Cann

Nicholas Carleton

Evan Carr

Olivia Carter

Kaitlin Casaletto

Luigi Castelli

Lahnna I. Catalino

Salvatore Catanzaro

Hannah Chang

Jennifer Cheavens

Nadia Chernyak

Arik Cheshin

Andrey Chetverikov

Louise Chim

Brian Chin

Michael Chmielewski

Margaret S. Clark

Patrick Clarke

Gerald L. Clore

John Coffey

Noga Cohen

Taya R. Cohen

Jeffrey F. Cohn

Karin G. Coifman

Daniel Cordaro

Nadia Corral-Frias

Joshua Correll

Kathleen Corrigall

Eduardo Coutinho

Florian Cova

L. Elizabeth Crawford

David Creswell

Carlos Crivelli

Jennifer Crocker

Damien Crone
Antonietta Curci

James Danckert

Wendy D'Andrea

Elise Dan-Glauser

Arnaud D'Argembeau

Mark H. Davis

Caroline Davis

Kelly D. Davis

Amy Dawel

Jasper de Groot

Jozefien De Leersnyder

Kim De Roover

Linda de Voogd

Jennifer Michele DeCicco

Andrew Delton

Heath A. Demaree

Kenneth G. DeMarree

Bryan Denny

Tom Denson

Anjolii Diaz

Stephan Dickert

Raymond DiGiuseppe

Anton Dijker

Daniel G. Dillon

Christian Dobel

Helen Dodd

Michael Dodd

Bruce Dore

Sylvie Droit-Volet

Peter Drummond

James Dungan

Elizabeth W. Dunn

Barney Dunn

David Dunning

Almudena Duque

Ulrich Ebner-Priemer

Andreas B. Eder

Boris Egloff

Hedwig Eisenbarth

Dan-Mikael Ellingsen

Robert A. Emmons

Yasemin I. Erbas

Thorsten M. Erle

Catherine Evers

Ariel Eytan

Allison Farrell

Mark Fedyk

Katya Fernandez

José Miguel Fernandez-Dols

Fernando Ferreira-Santos

Rebecca Ferrer

Emilio Ferrer-Caja

Eric A. Fertuck

Klaus Fiedler

Diana S. Fleischman
Luis E. Flores

Jaclyn Ford

Dan Foti

Jay Fournier

Mark G. Frank

Joe Franklin

Jon Freeman

Jennifer M.B. Fugate

Helene Fung

Shelly L. Gable

Brendan Gaesser

Sarah Gaither

Isaac Galatzer-Levy

Jody Ganiban

Nitika Garg

Agata Gasiorowska

Dylan Gee

Maria Gendron

Amy Gentzler

Laura Germine

Brandon E. Gibb

Cheryl Giddens

Kirsten Gilbert

Amanda C. Gingerich Hall

Yuthika Girme

Madeleine Goodkind

Amie M. Gordon

Stephanie Gorka

Reiko Graham

Margit Gramer

DeMond M. Grant

Kurt Gray

Katie Gray

Katharine Greenaway

Maud Grol

Sarah D. Gunnery

Melissa Hagan

Takeshi Hamamura

Stephan Hamann

Ian Handley

Benjamin L. Hankin

Kent Harber

Todd A. Hare

Shlomo Hareli

Eddie Harmon-Jones

Cindy Harmon-Jones

Neil Harrison

Elaine Hatfield

Katherine C. Haydon

James Heathers

Erin A. Heerey

Eric Hehman

Chelsea Helion

Scott H. Hemenover

Johannes Hewig

Clayton Hickey 
Joshua Hicks

Gilad Hirschberger

Edward R. Hirt

Hidefumi Hitokoto

Guy Hochman

Sara D. Hodges

Gordon Hodson

Berthold Hoecker

Katie Hoemann

Bernhard Hommel

$\mathrm{Xu}$ Hong

Kean Hsu

Kurt Hugenberg

Jeffrey R. Huntsinger

Almut Hupbach

William Ickes

Roland Imhoff

Tristen K. Inagaki

Frank J. Infurna

Alexandru Iordan

Rachael E. Jack Drew Jacoby-Senghor Jeremy Jamieson Julian Jara-Ettinger

Lisa Jaremka

Vikram Jaswal

Jessica Jenness

Xiaoming Jiang

Carly Johnco

Kareem Johnson

Jutta Joormann

Elise K. Kalokerinos

Philipp Kanske

Todd B. Kashdan

Nicholas Kelley

Shian-Ling Keng

Briana Kennedy

Pelin Kesebir

Yoav Kessler

Elizabeth J. Kiel

Chai-Youn Kim

Pilyoung Kim

B. Kyu Kim

Jungmeen Kim-Spoon

Laura A. King

Ulrich Kirk

Michael A. Kisley

Johanna Kissler

Shinobu Kitayama

Heide Klumpp

Lauren M. Knott

Leonie Koban

Julian Koenig

Birgit Koopmann-Holm

Sebastian Korb

Maria Kovacs

Philip Kragel

Mariska Kret

Ethan Kross

Anne-Wil Krujit

Eva G. Krumhuber
Roeline Kuijer

Amit Kumar

Janice Kuo

Kostadin Kushlev

Mikko Lähteenmäki

Jessica Lake

Brian Lakey

Jens Lange

Regina Lapate

Petri Laukka

Heidemarie Laurent

Sophie Lazarus

Julia Lee

Spike W. S. Lee

Bernhard Leidner

Brian Leitzke

Gert-Jan Lelieveld

Heather Lench

Sara M. Levens

Robert W. Levenson

Einat Levy-Gigi

Gary Lewis

Yaoran Li

Ye Li

Wang On Li

Belinda Liddell

Cesar F. Lima

Ottmar V. Lipp

Pan Liu

Kimberly M. Livingstone

Sander Los

Jessica Lougheed

Fantasy Lozada

Richard E. Lucas

Jennifer K. MacCormack

Dominique Maciejewski

Annmarie MacNamara

Matteo Malgaroli

Thomas Mann

Antony S. R. Manstead

Abigail A. Marsh

Amy Marshall

Aleix Martinez

Lynn Martire

Jennifer Mascaro

Takahiko Masuda

Jutta Mata

Andrew M. Mathews

Karen Mathewson

David Matsumoto

Bradley Mattan

Cynthia May

Michael McDermott

Jennifer McDermott

Dana L. McMakin

Kateri McRae

Harma Meffert

Brian P. Meier

Wendy Berry Mendes

Peter Mende-Siedlecki

Meghan Meyer

Katherine Mickley Steinmetz
Moira A. Mikolajczak

Eleanor Miles

Chiara Mirandola

Rani Moran

Sergio Morra

Marcello Mortillaro

Judith Moskowitz

Matt Motyl

Margaret Moulson

Erik Mueller

Amy Muise

Margaret Munger

Nora A. Murphy

Sandra Murray

Vishnu Murty

Keely Muscatell

Atsuko Nakagawa

Andrada Neacsiu

Rob M. A. Nelissen

Jackie Nelson

Charles A. Nelson

Nicole Nelson

Tamara Newton

Paula M. Niedenthal

Laura Niemi

Yael Nillni

Marret Noordewier

Ara Norenzayan

Greg Norman

Catherine J. Norris

Michael I. Norton

Lauri Nummenmaa

Jelena Obradovic

Aminda O'Hare

Hadas Okon-Singer

Sally Olderbak

Thomas M. Olino

Michael Olson

Anthony D. Ong

Keiichi Onoda

Suzanne Oosterwijk

Berge Osnes

Victor Ottati

Cristina Ottaviani

Christopher Oveis

Gewnhi Park

Brian Parkinson

Acacia Parks

Sona Patel

Indrajeet Patil

Megan Patterson

Wolfgang Pauli

Reinhard Pekrun

Marc D. Pell

Mikko Peltola

James Pennebaker

Luiz Pessoa

Jessica Peters

Erik Pettersson

Lauren Philbrook

Pierre Philippot
Jared Piazza

Paul Piff

Courtney Plante

Ruthie Pliskin

Richard S. Pond

Jesse L. Preston

Thomas F. Price

Jordan Quaglia

Sina Radke

Nairan Ramirez-Esparza

Rebecca E. Ready

Maija Reblin

Harry Reis

Rainer Reisenzein

Valerie Reyna

David Richter

Bernard Rimé

Ulrike Rimmele

Maureen Ritchey

Timothy D. Ritchie

Susan E. Rivers

Neil Roach

Oliver J. Robinson

Amy E. Root

Maya Rosen

Marcus Rothkirch

Joshua Rottman

Peter M. Ruberton

Jan Rummel

James A. Russell

Manuela Russo

Elske Salemink

Alejandro Salgado Montejo

Alvaro Sanchez

Henri Santos

Wataru Sato

Ajay Satpute

Donald Saucier

Alexandre Schaefer

Susanne Scheibe

E. Glenn Schellenberg

Jessica L. Schleider

Ralf Schmälzle

Julian Schmitz

Dominik Schoebi

Timothy P. Schofield

Jonathan Schooler

Lori Scott

Catherine Sebastian

Andrea Shafer

Golan Shahar

Amanda Shallcross

Stewart A. Shankman

Lisa B. Sheeber

Amitai Shenhav

Gal Sheppes

Michelle N. Shiota

Erika Siegel

Jen Silvers

Emiliana Simon-Thomas 
Elizabeth A. Simpson

Tamara Sims

Rafal Skiba

Richard B. Slatcher

Stephen D. Smith

Colin Smith

Leah H. Somerville

Gerri Spassova

Peggy St. Jacques

Sarah Stanton

Ursula M. Staudinger

Timo Stein

Michael Steinborn

Ryan Stolier

Viola Störmer

Daniel Stout

Timothy J. Strauman

Nina Strohminger

Daniel Strunk

Melissa Sturge-Apple

Jenny Su

Amy Summerville

Louise Sundararajan

Gaurav Suri

Michael Suvak

Cynthia Suveg

Kate Sweeny

Timothy Sweeny
Melanie Takarangi

Marco Tamietto

Maya Tamir

Jingzhi Tan

Patricia Tan

Kristina Tchalova

Jan Theeuwes

Ravi Thiruchselvam

Andrew Todd

Sascha Topolinski

Sheree Toth

Peter Totterdell

Jessica L. Tracy

Christopher Trentacosta

Ashlea Troth

Jeanne L. Tsai

Jo-Ann Tsang

Francis Tuerlinckx

Brianna Turner

Joshua Tybur

Christian Unkelbach

Heather L. Urry

Job van der Schalk

Lotte van Dillen

Florian van Leeuwen

Yvette Van Osch

Henk van Steenbergen
Jan W. van Strien

Wijnand A. P. Van Tilburg

Eric Vanman

Eduardo Vasquez

Ruut Veenhoven

Carlos Velasco

Philippe Verduyn

Alice Verstaen

Brian Vickers

Laura Visu-Petra

Bettina von Helversen

Dylan Wagner

Eric A. Walle

Sara Waters

Philip C. Watkins

Christian E. Waugh

Baldwin Way

Christian Webb

Thomas L. Webb

Aaron C. Weidman

Aaron Weidman

Anna Weinberg

Susanne Weis

Howard Weiss

Susan Wenze

Paul J. Whalen

Diana Whalen

Corey N. White
Sherri Widen

Matthias J. Wieser

Cynthia Willner

Tanja S. H. Wingenbach

Toby Wise

Sean Wojcik

Axel Wollmer

Janet Woodruff-Borden

Mary Woody

Jolie Wormwood

Maarten Wubben

Fei Xu

Akie Yanagi

Atefeh Yazdanparast

Dannii Yeung

K. Lira Yoon

Claire Zedelius

John Zelenski

Janice L. Zeman

Weiwei Zhang

Ruixue Zhaoyang

Christy Zhou Koval

David Ziegler

Peggy Zoccola

Ariel Zvielli 